\title{
A Day-ahead Centralized Unit Commitment Algorithm for A Multi-agent Smart Grid
}

\author{
Salam Hajjar \\ Universidade Federal do Rio de \\ Janeiro, COPPE, Ilha do fundo, \\ Rio de Janeiro, 21.945-970 RJ, \\ Brazil
}

\author{
Antoneta Iuliana Bratcu* \\ University of Grenoble Alpes \\ GIPSA-lab \\ Saint-Martin d'Heres, France
}

\author{
Ahmad Hably \\ University of Grenoble Alpes \\ GIPSA-lab \\ Saint-Martin d'Heres, France
}

\begin{abstract}
Renewable energy resources like wind and solar have become an effective factor in the energy production on the planet as they are inexhaustible renewable resources. However, they are very intermittent and their output cannot be predicted certainly. In this paper an algorithm of unit commitment within a power grid integrating wind and photovoltaic production units is proposed in a centralized approach that takes into account provisional data about the renewable energy production. Here the unit commitment problem is stated as a power demand coverage problem with some prespecified merit order list. A multi-agent architecture is proposed to facilitate the message exchange and easy addition and deletion of agents in the grid. This architecture is flexible and easy reconfigurable as it can provide solutions under assumptions of a decentralized approach. An implementation using JADE platform is presented in this work. The system is tested using real-data sets from an existent energy transport network in France (RTE). The results based on different operating conditions show the economic sense of the proposed strategy.
\end{abstract}

\section{INTRODUCTION}

The unit commitment (UC) problem aims at finding the least-cost dispatch of available generation resources to cover the load demand in an electrical grid. Production units' availability and setpoints are key decision issues in UC problem. Short Run Marginal Cost (SRMC), which is defined as the pure cost of electrical power production, is supposed to be the market-based price of electricity. However, costs of power generation evidently exceed this price, thus, power generators offer generation prices of their choice [1]. Obviously the power load depends strongly at each spacial zone on the period of the day and the season of the year. For example, in France a reasonably reliable demand forecast, as well as the list of available power units with their updated technical constraints and economic characteristics, are available each day by 4 p.m. Appropriate production schedules must then be computed, to be sent to the local units by 5 p.m. [2]. In the last years, renewable energy resources such as photovoltaic and wind turbine plants have been considered as a nonnegligible part of the energy offer since these resources are inexhaustible and their production cost is low compared to the conventional energy resources. However, uncertainty surrounds the availability of these renewable

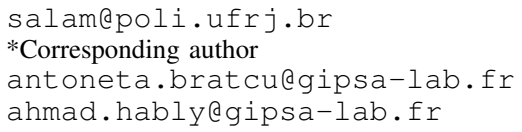

energy resources. The grid behavior becomes uncertain and the unit commitment problem must ensure the power demand covering without the need of a precise knowledge about the renewable energy units output.

A solid mathematical foundation has been built to help better understand the stochastic energy constraint and the inherent correlation between quality of energy and the uncertain energy supply [3]. Recently the domain of multiagent systems has achieved a real progress, it has been used to solve spatially-distributed and open problems. Power grids have a spatially distributed architecture and can be considered as systems made up of agents, some of which are energy producers, some other consumers acting on the energy market. For this, multi-agent architectures may be built to tackle different problems in power grids, such as the unit commitment problem.

In this paper, a centralized decision making algorithm is proposed to solve the UC problem at the level of an arbitrarily defined spacial geographical entity, region, or country, by using the advantage of a multi-agent architecture. The unit commitment problem is here focused on power demand coverage; to this end, it is based on a prespecified merit order list that can result from taking into account particularities of the considered geographical entity. The multi-agent architecture provides data exchange and avoids the problem of incompatibility between different types of software communicating agents. Assuming that agents are collaborative, the centralized decision provides a certain level of uniformity and coherence in controlling the grid.

This paper is organized as follows. Section II synthesizes some of related works. Section III shows the multi-agent architecture considered for the UC algorithm implementation. Section IV illustrates the proposed unit commitment algorithm. Section V discusses the results of applying the algorithm on real data scenarios. Finally, Section VII concludes the presented work and provides some perspectives.

\section{REVIEW OF RELATED WORK}

Decision making within power systems has been largely treated in the literature with different points of view. In [4], a distributed price-based control method is proposed. The controller takes its decision information from its neighbors. A day-ahead UC algorithm has been proposed in [5], where a decision making is achieved by a two-level decentralized 
framework. A low level treats each production unit individually to optimize its benefits. At the high level, coordinators communicate with their neighbors to update the prices by using a distributed method. In [6] energy storage systems (ESS) are studied as an alternative to provide flexibility in power system operation. The integration of a grid-scale ESS in short-term operational planning in a centralized costbased electricity market has suggested easier integration of renewable power resources as dispatchable units into a power generation grid. The rolling horizon strategy has been proposed in [7] as base of an energy management system for a renewable-energy-based micro-grid. In such strategy the control decision results iteratively from solving at each step a mixed-integer optimization problem. In order to provide on-line setpoints for each generation unit and signals for consumers based on a demand-side management mechanisms, forecasting models are used. This work treats globally the different agents for the benefit of the entire grid instead of concentrating on the individual benefit of each single producer.

An intelligent energy network system based on multi-agent systems has been proposed in [8]. It illustrates the advantage of using multi-agent structure over the centralized approach. Three types of agents - producers, loads and market contribute at solving the UC problem and providing the best setpoints planning according to information exchanged between the agents regarding the day-ahead consumption and production prevision. The work has been implemented on JADE multi-agent framework. This paper relies on the same idea, proposed in [8], of reducing the amount of information exchanged between agents. The two approaches differ at the level of computing the setpoints and treating the spot market.

\section{Multi-Agent ARChitecture}

Data exchange between agents may be simplified; thus, agents communicate between each other only the necessary information for the decision making. A multi-agent system is extendable open and open-ended system, i.e, agents can leave and new agents can join the system randomly, so that a theoretically infinite number of structures can be obtained. Dynamical behavior of agents makes that network management to be quite delicate process. Thus, some assumptions on the system can be adopted in order to facilitate the network's management. In this work two kinds of assumptions are made, which are categorized and listed as follows:

- Assumptions on the agents' behavior:

- Each agent must provide its status. If it is active, then its status is ON, otherwise it is OFF.

- An agent must maintain its status and the information exchanged with the other agents until the next cycle of data exchange.

- Agents are supposed to provide reliable information on their production, consumption, prices and costs.

- Assumptions on the grid architecture:

- The grid allows the integration of agents where the category is already recognizable by the grid. Agents of unknown or with undefined types will not be taken into account in the decision taking algorithm.

- The grid allows and does not limit the communication between its local agents. Agents can communicate with other agents out of the grid if assumptions on their behavior are respected.

The considered multi-agent grid is built up of five categories of agents representing the main actor types in a real power grid: (1) producer, (2) consumer, (3) storage unit, (4) external market and (5) controller or dispatcher. Since the focus is here on the decision-making process performed by the controller, the internal behavior of producers, consumers, storage units and external market is not detailed in the multiagent grid model considered in this work. Thus, agents are described mainly by their role on the market, as explained in the following set of assumptions about the energy market:

- Producer: This agent is an energy producer that offers its production on the market. Five types of producers are considered here: (1) Nuclear plant, (2) Hydraulic turbine, (3) Gas turbine, (4) Wind turbine plant and (5) Photovoltaic plant (PV). Any producer agent has a five-element profile: producer_type,energy_produced, price, starting_cost,state.

- Consumer: This agent represents any energy consumer which can be an industrial one, a residential one, etc. A consumer agent has a profile composed of only one element, consumption which represents energy to be consumed;

- Storage unit: This agent is an aggregated energy storage unit for the entire grid. This unit can store energy and/or provide energy to grid depending on the storage capacity and availability. This agent has a three-element profile: a limited storage_capacity, store_energy and a storage_cost. The store is considered unavailable if it is empty, i.e, the store_energy equals $0 \mathrm{MWh}$;

- External market: This agent represents an image of the market outside the considered grid. The grid can buy energy from the external market if the local producers cannot cover the consumers' needs. In this case, market can be seen as a source of energy. Extra energy produced locally can be sold to the external market instead of storing it, in case of financial benefit to the grid. It is supposed that external market can always absorb the extra energy. The decision to store the energy or sell it is taken by the UC algorithm. The market's profile consists of four elements: offered_energy, selling_price, purchasing_price, purchased_energy.

- Controller: This agent is responsible of providing a dayahead setpoint, planning of agents needed to cover the demand, prevision of potential extra energy production, decision whether to store or to sell the extra energy and the benefit of selling to external market. The controller profile has no elements since its aim is only to provide decisions upon running the UC algorithm based on data sent by the agents. 
Each agent has specific tasks to perform and a profile which determines its characteristics. The agent by itself can be seen as representing many instances of its type. Agents communicate with each other through messages which contain only the necessary information for taking the UC decision. These messages in their simplest form contain only the values of the agent profiles' elements. The decision is taken in a centralized manner by the controller. The controller is assumed to be a trusted agent; thus, other agents can communicate to the controller true values of consumption, production and prices. The relation between all agents is represented in Figure 1.

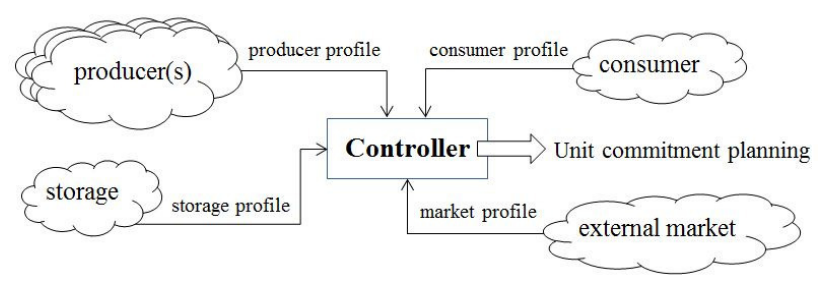

Fig. 1. Smart grid communicating agents.

\section{UNIT COMMITMENT ALGORITHM}

In this section a day-ahead unit commitment algorithm with a decision horizon of 48 periods ( 30 minutes each) in the day-ahead market is considered. The algorithm has two main goals: (1) to cover the consumption by the local production using the most trustful agents available; in this way consumer needs can be met through the offers proposed by the most available producers who make the most interesting offers; (2) to maximize the production benefit by selling the extra produced energy, if any, to the external market.

\section{A. Assumptions}

In order to satisfy the consumption needs, the controller has direct connection with producers, storage and the external market. Producers, consumers and storage send messages to the controller regarding their production, consumption needs and offers information, respectively. The controller decides, depending on the received information, which producers must be called to cover the consumer needs and in which order. Dispatching order will depend on producers dispatchability and size. From this point of view, renewable energy units have low dispatchability because of their intermittence and unpredictability. Therefore, they will not be sollicited as main producers, but as so-called backup units. Among the different types of producers mentioned in Section III studies show that the nuclear plants come in first place in energy production as their production is fully dispatchable and may reach thousands of MW depending on the plant size. For example, the production of the nuclear central in Rhône-Alpes region in France can range from 4000 MWh to $12000 \mathrm{MWh}$. Hydraulic turbines come in the second place as they can produce energy that ranges from $1000 \mathrm{MWh}$ to $4000 \mathrm{MWh}$. Gas turbines come in the third place as their production reaches only to few hundreds of MW. Wind and photovoltaic plants have the lowest sollicitation level as their production capacity is strongly related to the season of the year, the hour of the day and the geographic location

In order to propose a day-ahead planning for the agents in the system, the controller, at each sampling period, calls the available agents whose status is ON. The algorithm calls first the fully dispatchable production units, then the backup ones. At first place it calls the nuclear producer with the entire amount of produced energy. It compares the consumption demand with the energy produced by the nuclear agent. If the nuclear agent cannot cover alone the demand, then the algorithm calls the hydraulic turbine agent. It calculates the sum of the energy produced and compares it to the consumption demand. If the demand remains unsatisfied at this point, the controller calls the storage. Since the storage does not have a starting cost it becomes practically cheaper than the gas turbine producer. The controller continues calling agents and check power balance. It calls the gas turbine agent, then the PV agent and finally the wind turbine agent. It is considered judicious for the PV plants to come in priority before the wind turbine agent since PV plants are cheaper to start, although they are not available the whole day. Such choice is confirmed in [9]. At each step, if the accumulated value of produced energy exceeds the consumption demand, the controller stops calling agents and calculates the amount of extra energy as follows:

extra_energy $=$ accumulated_energy - consumption (1)

In case extra energy is produced the controller decides either to store this energy or to sell it to the external market. The decision to sell or store relies on the following reasoning:

$$
\begin{aligned}
& \text { If }(\text { extra_energy } \times \text { market_selling_price }(p))> \\
& {[\text { extra_energy } \times \text { market_selling_price }(p+1)-} \\
& \quad \text { extra_energy } \times \text { stock_cost }(p)]
\end{aligned}
$$

then sell the extra_energy at period $p$

else store the amount of energy regarding the storage capacity to be sold at period $p+1$ and sell the remaining at period $p$

Since this decision is strongly dependent on the external market price, it is quite difficult to predict it, as the external market price oscillates depending on the spot market price.

\section{B. Decision-making strategy}

The 10-step algorithm flowchart is given in Figure 2. At step 1, the controller collects the required data and reads the messages sent by the available agents in the network. At step 2, the controller initializes two lists: (1) a list "available_agents", which contains the activated agents with their profile values, (2) the list "called_agents", initially empty, which will contain, by the end of the algorithm, the group of agents which satisfy together the consumption demand. In case of an absent agent, its profile will be replaced by null in the list and values of its elements will be replaced by zeros. At step 3, the agents in the 
list "available_agents" are sorted, by type. Each agent is given a priority degree, so that the controller calls the agents in their order. At step 4, the controller verifies if the consumption demand is satisfied. Obviously, at the first cycle of the algorithm the demand would not be satisfied, since no agents are yet called. At step 5, the controller calls an agent from the list "available_agents" according to the priority order. At step 6, the controller updates the list of called agents. At step 7, the satisfied demand is updated depending on the energy offered by the last called agent. This update is computed as follows:

satisfied_demand $:=$ satisfied_demand + agent_energy

At step 9 the extra energy production is computed as follows:

$$
\text { extra_energy }:=\text { consumption - satisfied_demand }
$$

If extra energy is produced then the controller goes to step 9, otherwise, the algorithm returns to step 4, continues towards the end and provides the proposed UC planning. At step 9, the decision whether to sell the extra energy at the current period $p$ or to store it, is taken knowing that the storage agent has a limited capacity, it is not sure that the entire extra energy can be stored. If it cannot be stored, extra energy is sold to the external market at period $p$ whatever its financial income is. The algorithm arrives to its end at step 10 , which is reachable either from step 4 or from step 9. If the end is reached from step 4, this means that the exact consumption amount can be produced by the called agents and no financial gain is obtained. When the end is reached from step 9, an extra energy has been produced and some financial gain is earned. At step 10, the UC plan is proposed for the period $p$. It consists of a list of called agents with a detailed report illustrating the consumption demand, the production that covered the demand, the amount to be paid to the called agents, which is the sum of their individual prices, the decision taken about the selling to the external market or storing and the selling income. It is supposed that the controller requires from the storage unit only the needed amount of energy to cover the demand. Thus, at any period $p$, if the storage contains the energy needed to cover the consumption, then no extra energy is necessary.

\section{CAse studies}

The proposed UC algorithm is applied on two case studies. The first one represents the consumption and production in a normal year day in France, the $15^{\text {th }}$ of May 2014. The second is $25^{t h}$ of December 2013, a winter day during holidays where the consumption is supposed to be different from typicall. The day-ahead consumption and production prevision data is picked up for the Rhône-Alpes and Provence-AlpesCôte-d'Azur from the website of French electricity transport grid (Réseau du transport d'électricité - RTE, http://www.rtefrance.com/en/eco2mix/eco2mix-donnees-regionales-en).

\section{A. JADE implementation model}

In this work JADE (Java Agent Development Framework) platform [10] is employed to implement the UC algorithm.

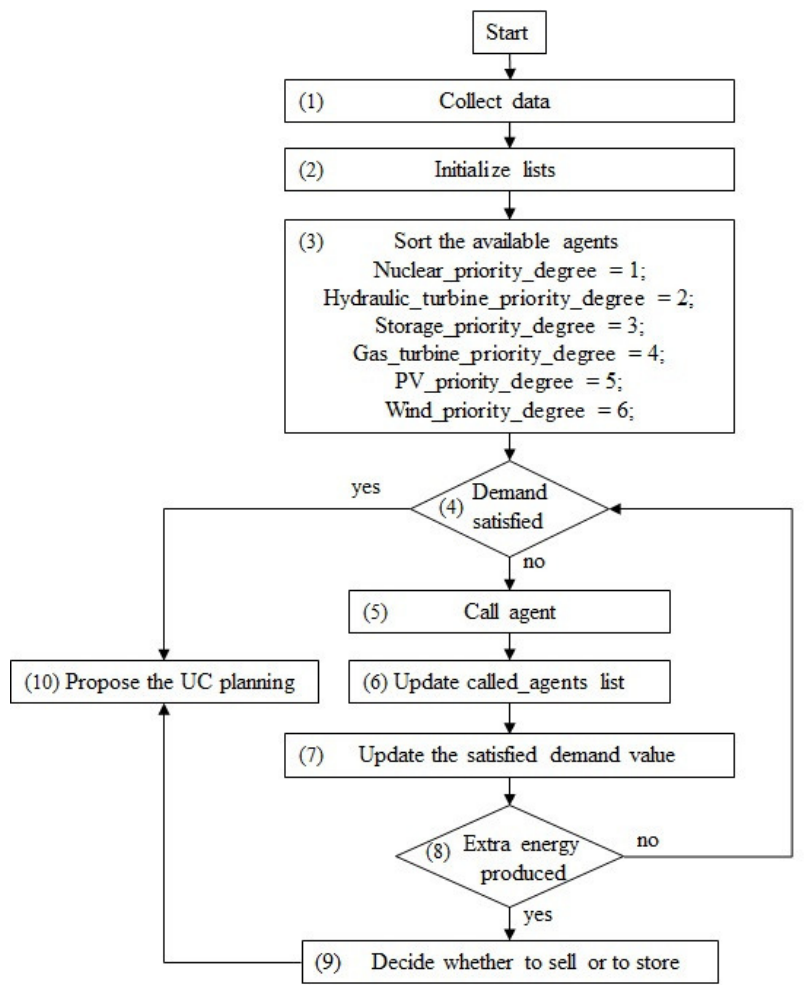

Fig. 2. Overview of decision-making flowchart.

JADE is a programming framework that significantly facilitates the implementation of agent-based applications in compliance with the FIPA ${ }^{1}$ specifications. Five producer agents (nuclear, hydraulic turbine, gas turbine, wind and PV), a grid-level storage unit, a consumer which represents the aggregated consumption of the entire grid in the considered region and the external market are modeled by software agents. The behavior of each agent is described by a set of arrays containing the values of the agent profile's elements. The controller agent is a decision-making software agent who runs the UC algorithm explained in section IV. The agents - producers, consumer, external market and storage - send messages to the controller containing the integer values of their individual profiles, i.e, the previsions of production, consumption and prices for the next day. The controller computes the setpoints for 48 time intervals and proposes the agents' commitment planning for the next day.

\section{B. Discussion of results}

For May $15^{\text {th }} 2014$ scenario Figure 3 illustrates the energy production predicted for PV plants, wind turbine plants and gas turbines one day ahead. Wind power prevision is based on the slowly variable, seasonal component of wind speed, so a-half-an-hour sampling time is sufficient for good prediction accuracy. Note that the production value of these producers ranges from $0 \mathrm{MWh}$ to $220 \mathrm{MWh}$ at its highest value produced by the PV agent at its peak of production.

${ }^{1}$ Foundation of Intelligent Physical Agents 


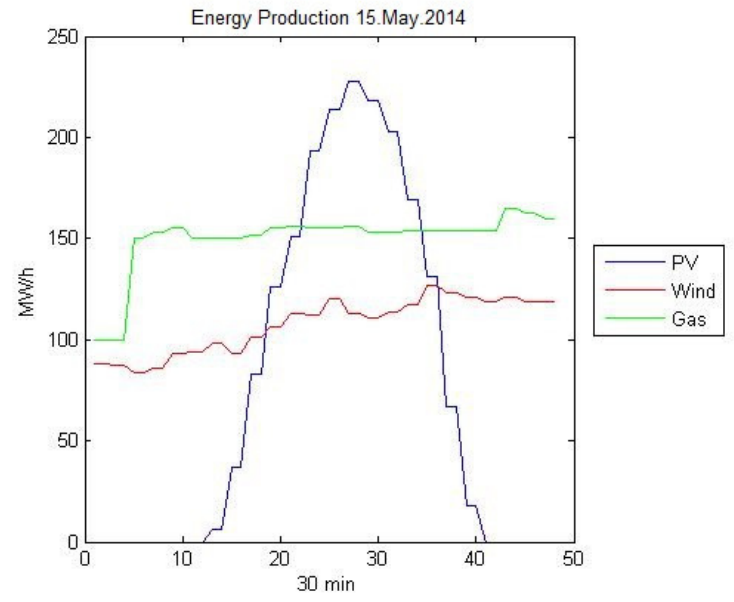

Fig. 3. PV, wind, and gas production prevision for May 15th 2014.

Figure 4 illustrates prevision of consumption needs and production of the different types of producers. PV, wind and gas turbine productions have been represented as an aggregated curve called backup energy. Logarithmic scale in $y$ axis is preferred since the backup energy is much smaller compared to those of other agents. The consumption at that day ranged from $10500 \mathrm{MWh}$ to $14600 \mathrm{MWh}$. Note that production of nuclear and hydraulic turbine agents is much higher than production of the gas turbine, PV and wind agents. The UC algorithm has been applied to this

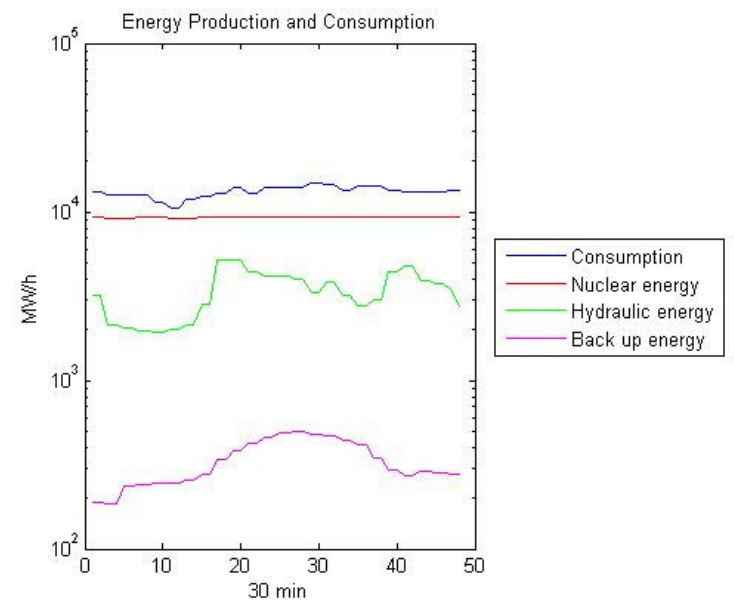

Fig. 4. Consumption and production prevision for May 15th 2014, logarithmic scale.

consumption and production scenario. Figure 5 illustrates the consumption cover realized by the consumers, and the extra energy produced at periods $p_{i}$ where ${ }^{2} i=\{[8-11],[14-$ $25],[38-45]\}$. Here also, the backup energy cummulates $\mathrm{PV}$, wind and gas turbine productions and $y$ axis values are in logarithmic scale. A zoomed view over the extra energy produced is shown in Figure 6. The algorithm decides

${ }^{2}$ from period 8 to 11 , from period 14 to 25 and from period 38 to 45 according to (2) and the market price evolution in Figure 7 whether to sell the extra energy at period $i$ or to store it. The algorithm indicates for the scenario of $15^{\text {th }}$ of May that the immediate selling of extra energy is beneficial compared to storing the energy. Computation of the selling income shows that it reaches its highest value at periods 18 and 19, which correspond to the peak of market price and the peak of extra energy production. However, this prevision of income cannot be taken as systematically predictable behavior since neither the market price, nor the extra energy production are constant or systematically repetitive.

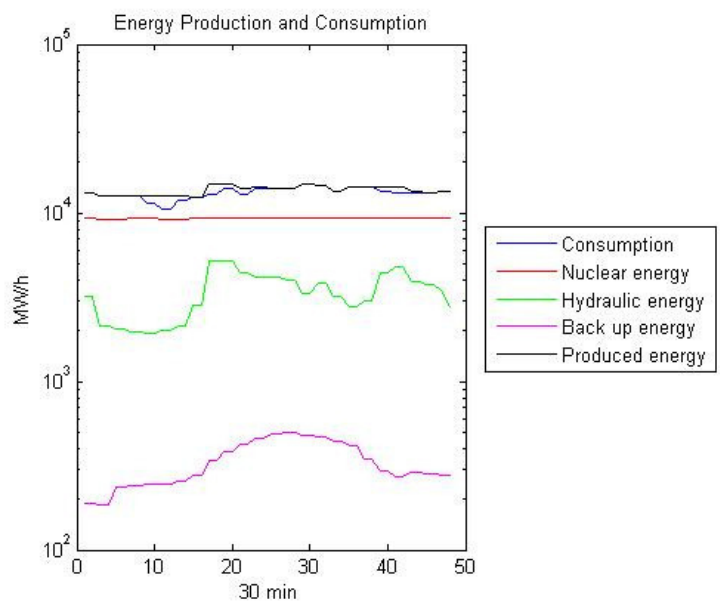

Fig. 5. Consumption needs covering on May 15th 2014, logarithmic scale.

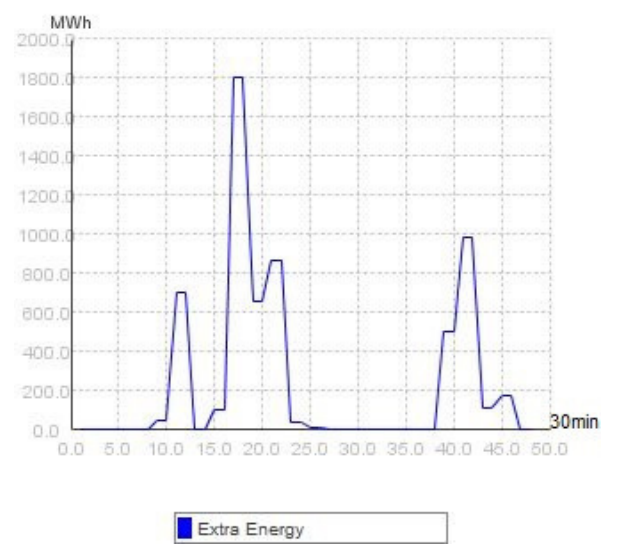

Fig. 6. Extra energy produced on May 15th 2014.

\section{Results comparison}

We focus in this discussion on the effect of the PV and wind energy resources on the grid. We argue the financial advantage of integration of these resources in a large-scale grid such as the one considered in this work. PV and wind production prevision data for the $25^{\text {th }}$ of December 2013 are shown in Figure 8. Note that the PV production peak dramatically decreased from $210 \mathrm{MWh}$ in May to $32 \mathrm{MWh}$ in December. Similarly the wind production peak decreased 


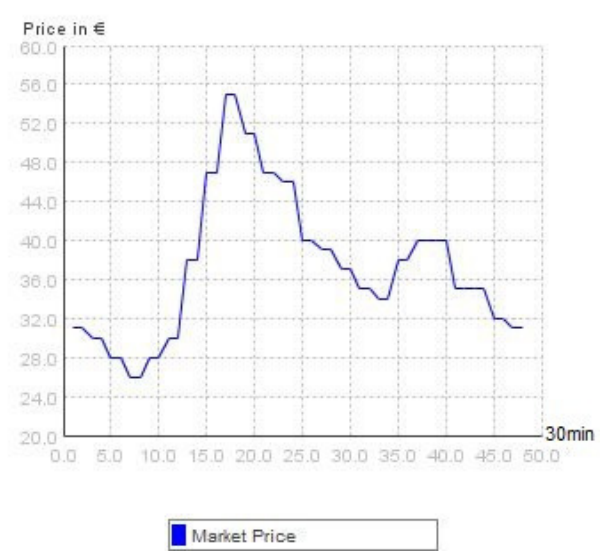

Fig. 7. Spot market price on May 15th 2014.

from $163 \mathrm{MWh}$ in May to $82 \mathrm{MWh}$ in December. The algorithm results on the scenario of $25^{\text {th }}$ of December, illustrated in Figure 9, show on one hand that the considered UC algorithm achieves covering the consumers' needs by calling the external market at most of the day time. The backup energy cummulates PV, wind and gas turbine productions and $y$ axis values are in logarithmic scale. On the other hand, a very small amount of extra energy is produced at periods 10 and 25 which are the peak periods of wind and PV, respectively. This confirms that the renewable energy plants can be integrated into the power grid as backup agents. When their energy is not necessary to cover the power demand, it can be sold to the external market. Practically this might be much effective financially in Spring than in Winter.

Indeed, the considered context - characterized by use with predilection of nuclear energy - render the renewable energy sources dependent on the external market price. This situation does not foster development of renewable small-power sources as grid units, unless they are made fully dispatchable. To this end, a possibility is their association with storage units and implementation of an effective local power flow management.

\section{SCALABILITy AND PERFormance}

The scalability of the proposed algorithm depends on several factors: the messages exchange time, the size of data within messages and the number of communicating agents. To evaluate the scalability of the platform, one can start with a small number of communicating agents, for example, the controller, the customer and two power providers, then enlarge the network by increasing the number of power providers. One can note that the computation time grows when the number of network agents increases. This results from the increase in the number of messages exchanged between agents and the time needed to extract the data out of the messages. The performance of the algorithm depends strongly on the implementation environment, i.e, the performance of each agent's machine and the network speed. In this work, the used implementation environment is one Dell personal computer, where Jade 3.2 and Eclipse

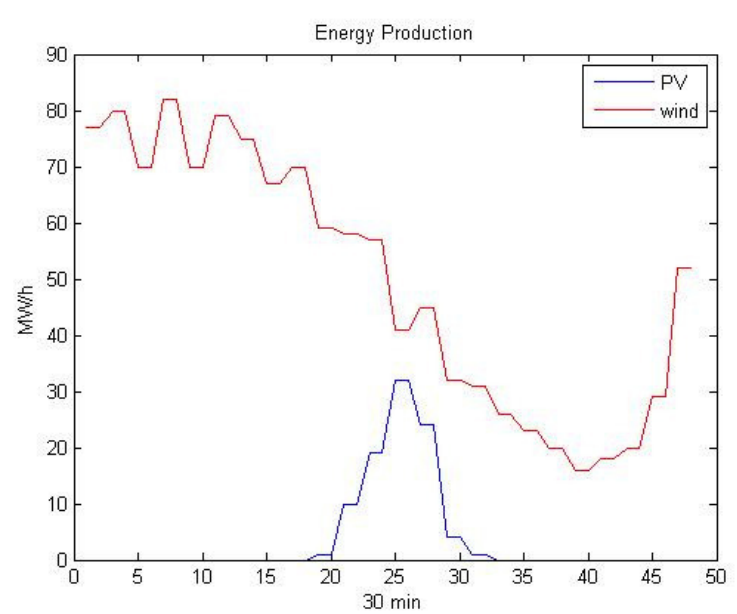

Fig. 8. Wind and PV produced energy on December 25th 2013.

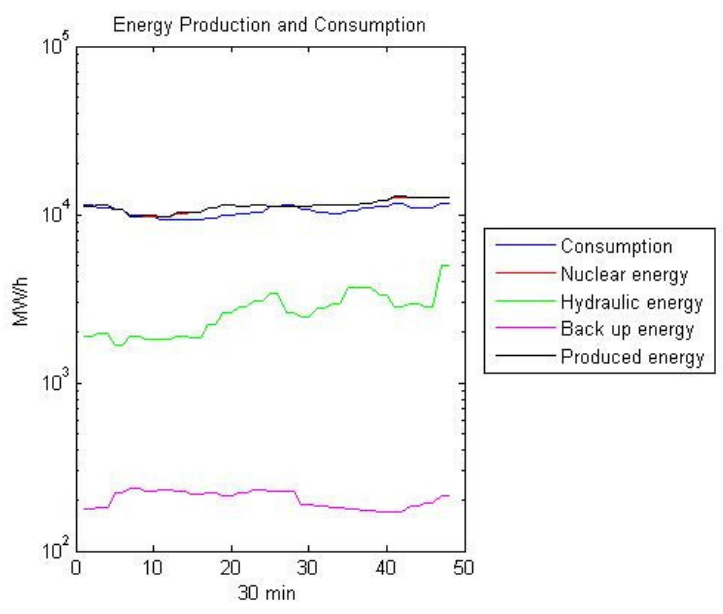

Fig. 9. Consumption covering on December 25th 2013, logarithmic scale.

IDE for Java developers are installed. The algorithm has been run off-line since JADE platform played the role of multiagent network. Thus, the computer characteristics strongly influence the algorithm performance. We indicate here that the run time of the studied example was about 0.5 seconds. Table I provides the characteristics of used PC.

\section{CONCLUSION}

The work presented in this paper proposes a centralized unit commitment algorithm, implemented on a multi-agent architecture. The algorithm achieves to cover the demand of consumers in a power grid by taking into account dailyvariation-based previsions of renewable energy production, mainly PV and wind turbines. It illustrates the advantages of using a multi-agent architecture in simplifying the exchange of messages between agents. The proposed algorithm is applied to real data retrieved in Rhône-Alpes and ProvenceAlpes-Côte d'Azur in France. Analyzing how the proposed algorithm optimizes the schedule of power plants with respect to total cost is a further issue. Such analysis may be 
TABLE I

Implementation Machine's Characteristics

$\begin{array}{ll}\text { Model } & \text { Dell Inspiron } \\ \text { Processor } & \text { Intel core i5 CPU M560@2.67 GHz x } 4 \\ \text { Total memory } & 3.7 \text { GB } \\ \text { Operating System } & \text { 64-bit Debian } \\ \text { OS version } & 7.3 \text { (Wheezy) } \\ \text { JADE version } & 3.2 \\ \text { Eclipse version } & \text { IDE for Java developers } \\ \text { JAVA } & \text { Sun SDK } 1.4\end{array}$

useful to assess effectiveness against other methods for solving the unit commitment problem in its classical formulation. The proposed multi-agent architecture is flexible to accommodate different centralized decision making strategies. This architecture can easily be modified to accommodate more complex agent scenarios and agent interaction mechanisms, energy market mechanisms and decision-making strategies, in order to put into light different patterns of behavior within a decentralized context, that are difficult to predict.

\section{ACKNOWLEDGMENT}

This work was achieved in the framework of the Smart Energy project funded by Grenoble Institute of Technology in Grenoble, France, to whom authors are kindly grateful.

\section{REFERENCES}

[1] M. Lively, "Short run marginal cost pricing for fast responses on the smart grid," in Procs. of 2010 Innovative Smart Grid Technologies, January 2010, pp. 1-6, DOI 10.1109/ISGT.2010.5 434779.

[2] L. Dubost, R. Gonzalez, and C. Lemar' echal, "A primal-proximal heuristic applied to the french unit-commitment problem," Math. Program., vol. 104(1), pp. 129-151, 2005, DOI 10.1007/s10107-0050593-4.

[3] W. Kui, J. Yuming, and D. Marinakis, "A stochastic calculus for network systems with renewable energy sources," in Procs. of 2012 IEEE Conference on Computer Communication Workshops, March 2012, pp. 109-114, DOI 10.1109/INFCOMW.2012.6 193470.

[4] A. Joki' c, M. Lazar, and P. van den Bosch, "Price-based control of electrical power systems," in Intelligent Systems, Control and Automation: Science and Engineering (vol. 92 Intelligent Infrastructures), R. R. Negenborn and Z. Lukszo and H. Hellendoorn (Eds.), 2010, pp. 109-131, DOI 10.1007/978-90-481-3598-1 5.

[5] L. Mingyang and P. Luh, "A decentralized framework of unit commitment for future power markets," in Procs. of 2013 IEEE Power and Energy General Meeting, July 2013, pp. 1-5, DOI 10.1109/PESMG.2013.6 672790.

[6] C. Suazo-Martinez, E. Pereira-Bonvallet, R. Palma-Behnke, and X.-P. Zhang, "Impacts of energy storage on short term operation planning under centralized spot markets," IEEE Trans. on Smart Grid, vol. 5(2), pp. 1110-1118, 2014, DOI 10.1109/TSG.2013. 2281828.

[7] R. Palma-Behnke, C. Benavides, F. Lanas, B. Severino, L. Reyes, J. Llanos, and D. Saez, "A microgrid energy management system based on the rolling horizon strategy," IEEE Trans. on Smart Grid, vol. 4(2), pp. 996-1006, 2013, DOI 10.1109/TSG.2012.2231440.

[8] S. Abras, C. Ki' eny, S. Ploix, and F. Wurtz, "Mas architecture for energy management: Developing smart networks with jade platform," in Procs. of 2013 IEEE Intl. Conference on Smart Instrumentation, Measurement and Applications, November 2013, pp. 1-6, DOI 10.1109/ICSIMA.2013.6 717913.

[9] M. Zipf and D. Most, "Impacts of volatile and uncertain renewable energy sources on the german electricity system," in Procs. Of 2013 10th Intl. Conference on the European Energy Market, May 2013, pp. 1-8, DOI 10.1109/EEM.2013.6 607397.

[10] F. Bellifemine, G. Caire, and D. Greenwood, Developing Multiagent Systems with JADE. New York, USA: Wiley, 2007. DOI 10.1002/ 9780470058411. 